\title{
183.
}

\section{NOTE ON CERTAIN SYSTEMS OF CIRCLES.}

\section{[From the Quarterly Mathematical Journal, vol. II. (1858), pp. 83-88.]}

IT will be convenient to remark at the outset that two concentric circles, the radii of which are in the ratio of $1: i$ ( $i$ being as usual the imaginary unit), are orthotomic $\left({ }^{1}\right)$, and that the most convenient quasi representation of a circle, the centre of which is real and the radius a pure imaginary quantity, is by means of the concentric orthotomic circle. This being premised consider a circle and a point $C$. The points of contact of the tangents through $C$ to the circle may be termed the taction points; the points where the chord through $C$ perpendicular to the line joining $C$ with the centre meets the circle, may be termed the section points. It is clear that, for an exterior point, the taction points are real and the section points imaginary, while, for an interior point, the section points are real and the taction points imaginary. A circle having $C$ for its centre and passing through the taction points (in fact the orthotomic circle having $C$ for its centre) is said to be the taction circle. A circle having $C$ for its centre and passing through the section points is said to be the section circle. Of course for an exterior point the taction circle is real and the section circle imaginary; while for an interior point the taction circle is imaginary and the section circle is real. It is proper also to remark that the taction circle and the section circle are concentric orthotomic circles.

Passing now to the case of two systems of orthotomic circles, let $M M^{\prime}, N N^{\prime}$ be lines at right angles to each other intersecting in $R$, and let $M, M^{\prime}$ be real or pure imaginary points on the line $M M^{\prime}$, equidistant from $R$. Imagine a system of circles,

1 Two concentric circles are, it is well known, conics having a double contact at infinity, and it appears at first sight difficult to reconcile with this, the idea of two particular concentric circles being orthotomic. The explanation is that any two lines through a circular point at infinity may be considered as being at right angles to each other, and therefore any line through a circular point at infinity may be considered as being at right angles to itself. The two concentric eircles in question have, in fact, at each circular point at infinity a common tangent, but this common tangent must be considered as being at right angles to itself. The paradox disappears entirely upon a homographic deformation of the figure; two lines $K L$, $K M$ are then defined to be at right angles when joining $K$ with the fixed points $I, J$, the four lines $K L$, $K M, K I, K J$ are a harmonic pencil; but when $K$ coincides with $I$, then $K I$ is indeterminate and may be taken to be the fourth harmonic of the pencil, i.e. any two lines $I L, I M$ through the point $I$ may be considered as being at right angles. 
each of them having its centre on the line $N N^{\prime}$ and passing through the points $M, M^{\prime}$ (so that $M M^{\prime}$ is the radical axis of these circles). There are always on the line $N N^{\prime}$ two pure imaginary or real points $N, N^{\prime}$ equidistant from $R$, such that the circles, each of them having its centre on $M M^{\prime}$ and passing through the points $N, N^{\prime}$ ( $N N^{\prime}$ being therefore the radical axis of these circles), are orthotomic to the first-mentioned system of circles, [the four points $M, M^{\prime}, N, N^{\prime}$ being thus as I have since termed them, two pairs of antipoints]. Moreover if $R$ be made the centre of a circle passing through $M, M^{\prime}$, then the concentric orthotomic circle passes through $N, N^{\prime}$; this is in fact only a particular case of the general property.

Suppose now that $M, M^{\prime}$ being given as the points of intersection of two circles having their centres on $N N^{\prime}$, it is required to find a circle having for its centre a given point $C$ on $N N^{\prime}$ and passing through the points $M, M^{\prime}$. In the case of $M, M^{\prime}$ being real, the required circle is obviously given and is always real. But if $M, M^{\prime}$ are imaginary; then if about any point of $M M^{\prime}$ as centre a circle be described orthotomic to one of the circles, it will be orthotomic to the other circle, and will meet $N N^{\prime}$ in the real points $N, N^{\prime}$. Now if $\rho$ be the radius of the required circle (i.e. of the circle having $C$ for its centre and passing through the points $M, M^{\prime}$ ), then $\rho^{2}=(R C)^{2}+(R M)^{2}=(R C)^{2}-(R N)^{2}$. Hence if $R C>R N$ or if $C$ lies outside the space $N N^{\prime}, \rho^{2}$ is positive or the required circle is real, and the radius is at once constructed from the preceding expression

$$
\rho^{2}=R C^{2}-R N^{2} .
$$

But if $R C<R N$ or $C$ lies within the space $N N^{\prime}$, then the required circle is imaginary, but the concentric orthotomic circle is at once constructed from the formula

$$
\rho^{\prime 2}=R N^{2}-R C^{2}
$$

Suppose now the point $C$ is a centre of similitude of the two circles. The circle having $C$ for its centre and passing through the points $M, M^{\prime}$ is a taction circle of all the taction circles of the two circles, it may be termed the tactaction circle. The concentric orthotomic of the circle having $C$ for its centre and passing through the points $M, M^{\prime}$ is a section circle of all the taction circles of the two circles, it may be termed the sectaction circle. Consider first the case where the circles intersect in a pair of real points; here the two centres of similitude are on opposite sides of $R$; the tactaction circles are both real, the sectaction circles both imaginary. Secondly, the case where the two circles are wholly exterior each to the other, the two centres of similitude lie on the same side of $R$, viz. the centre of inverse similitude between $R$ and $N$, the centre of direct similitude beyond $N$. Hence the tactaction circle corresponding to the centre of direct similitude and the sectaction circle corresponding to the centre of inverse similitude are real, the other tactaction circle and sectaction circle are imaginary. Thirdly, the case where one of the circles is wholly interior to the other; here the two centres of similitude are still on the same side of $R$, but the centre of direct similitude lies between $R$ and $N$, and the centre of inverse similitude lies beyond $N$. Hence the sectaction circle corresponding to the centre of direct similitude and the tactaction circle corresponding to the centre of inverse similitude are real, the other sectaction circle and tactaction circle are imaginary. 
To obtain a distinct idea of the methods made use of in Gaultier's "Mémoire sur les moyens généraux de construire graphiquement un cercle déterminé par trois conditions," (Jour. École Polyt. t. Ix. [1813], p. 124), and in Steiner's "Geometrische Betrachtungen," Crelle, t. I. [1826], p. 161; it should be remarked that both of these geometers, confining as they do their attention to real circles, do not consider the section circle of an exterior point, or the taction circle of an interior point. The taction circle of an exterior point, or the section circle of an interior point, is Gaultier's "Cercle radical," and Steiner's "Potenzkreis," and Steiner also speaks of the radius of this circle as the "Potenz" of its centre in relation to the given circle. The nature of the Cercle radical, or Potenzkreis, (i.e. whether it is a taction circle or a section circle) is of course determined as soon as it is known whether the centre is an exterior or an interior point, and Gaultier distinguishes the two cases as the "radical réciproque" and the "radical simple," and in like manner Steiner speaks of the Potenz as being "äuszerlich" or "innerlich." Again, for two circles and for a given centre of similitude Gaultier and Steiner employ the tactaction circle or the sectaction circle, whichever of them is real, Gaultier without giving any distinctive appellation to the circle in question, Steiner calling it the Potenzkreis of the two circles, and in particular the "äuszere Potenzkreis" or the "innere Potenzkreis," according as it has for centre the centre of direct similitude or the centre of inverse similitude.

The preceding properties of circles are of course at once extended to conics passing each of them through the same two points; it is I think worth while to notice what the analogue is of a pair of concentric orthotomic circles. If the fixed points are $I, J$ and if the point corresponding to the centre is $K$, then the conics are of course conics touching the lines $K I, K J$ in the points $I, J$, and, one of the conics being given, the other is to be determined. It is easily seen that if an arbitrary line through $I$ meets the conics in $P, P^{\prime}$ and the line $K J$ in $M$, then the points $I, M, P, P^{\prime}$ are a harmonic range, and this condition gives the construction of the second conic; it of course follows that an arbitrary line through $J$ meets the conics in points $Q, Q^{\prime}$ and the line $K I$ in a point $N$ such that the points $J, N, Q, Q^{\prime}$ are also a harmonic range. The two conics in question may be termed "inscribed harmonics" each of the other.

Addition. The equation of the tactaction circle, corresponding to the centre of direct (or inverse) similitude, of two given circles, may be found as follows:

Let the equations of the given circles be

$$
\begin{aligned}
& (x-\alpha)^{2}+(y-\beta)^{2}=c^{2}, \\
& \left(x-\alpha^{\prime}\right)^{2}+\left(y-\beta^{\prime}\right)^{2}=c^{\prime 2},
\end{aligned}
$$

then the coordinates of the centre of direct similitude are

$$
\frac{a c^{\prime}-\alpha^{\prime} c}{c^{\prime}-c^{\prime}}, \frac{\beta c^{\prime}-\beta^{\prime} c}{c^{\prime}-c}
$$

which are therefore the coordinates of the centre of the tactaction circle; and the equation of this circle is of the form

$$
\lambda\left[(x-\alpha)^{2}+(y-\beta)^{2}-c^{2}\right]+(1-\lambda)\left[\left(x-\alpha^{\prime}\right)^{2}+\left(y-\beta^{\prime}\right)^{2}-c^{\prime 2}\right]=0,
$$

C. III. 
or, expanding and reducing,

$\left(x^{2}+y^{2}\right)-2\left[\alpha \lambda+\alpha^{\prime}(1-\lambda)\right] x-2\left[\beta \lambda+\beta^{\prime}(1-\lambda)\right] y+\lambda\left(\alpha^{2}+\beta^{2}-c^{2}\right)+(1-\lambda)\left(\alpha^{\prime 2}+\beta^{\prime 2}-c^{\prime 2}\right)=0$.

We must therefore have

$$
\begin{aligned}
& \alpha \lambda+\alpha^{\prime}(1-\lambda)=\frac{\alpha c^{\prime}-\alpha^{\prime} c}{c^{\prime}-c}, \\
& \beta \lambda+\beta^{\prime}(1-\lambda)=\frac{\beta c^{\prime}-\beta^{\prime} c}{c^{\prime}-c},
\end{aligned}
$$

which are consistent with each other and give

$$
\lambda=\frac{c^{\prime}}{c^{\prime}-c}, \quad 1-\lambda=\frac{-c}{c^{\prime}-c}
$$

we have then

$$
\lambda\left(\alpha^{2}+\beta^{2}-c^{2}\right)+(1-\lambda)\left(\alpha^{\prime 2}+\beta^{\prime 2}-c^{\prime 2}\right)=\frac{1}{c^{\prime}-c}\left[c^{\prime}\left(\alpha^{2}+\beta^{2}\right)-c\left(\alpha^{\prime 2}+\beta^{\prime 2}\right)+c c^{\prime}\left(c^{\prime}-c\right)\right] ;
$$

and the equation of the tactaction circle is

$$
x^{2}+y^{2}-2 \frac{\alpha c^{\prime}-\alpha^{\prime} c}{c^{\prime}-c} x-2 \frac{\beta c^{\prime}-\beta^{\prime} c}{c^{\prime}-c} y=\frac{1}{c^{\prime}-c}\left[c^{\prime}\left(\alpha^{2}+\beta^{2}\right)-c\left(\alpha^{\prime 2}+\beta^{\prime 2}\right)+c c^{\prime}\left(c^{\prime}-c\right)\right],
$$

which may also be written

$$
\left(x-\frac{\alpha c^{\prime}-\alpha^{\prime} c}{c^{\prime}-c}\right)^{2}+\left(y-\frac{\beta c^{\prime}-\beta^{\prime} c}{c^{\prime}-c}\right)^{2}=\frac{c c^{\prime}}{\left(c^{\prime}-c\right)^{2}}\left[\left(\alpha^{\prime}-\alpha\right)^{2}+\left(\beta^{\prime}-\beta\right)^{2}-\left(c^{\prime}-c\right)^{2}\right] .
$$

We have thus the equation of the tactaction circle corresponding to the centre of direct similitude, and that of the tactaction circle corresponding to the centre of inverse similitude is at once obtained from it by changing the sign of one of the two radii $c, c^{\prime}$.

Consider any three circles and combining them in pairs, by what has preceded the equations of the tactaction circles corresponding to the centres of direct similitude will be

$$
\begin{gathered}
\left(c^{\prime \prime}-c^{\prime}\right)\left(x^{2}+y^{2}\right)-2\left(\alpha^{\prime} c^{\prime \prime}-\alpha^{\prime \prime} c^{\prime}\right) x-2\left(\beta^{\prime} c^{\prime \prime}-\beta^{\prime \prime} c^{\prime}\right) y \\
+c^{\prime \prime}\left(\alpha^{\prime 2}+\beta^{\prime 2}\right)-c^{\prime}\left(\alpha^{\prime \prime 2}+\beta^{\prime \prime 2}\right)+c^{\prime} c^{\prime \prime}\left(c^{\prime \prime}-c^{\prime}\right)=0 \\
\left(c-c^{\prime \prime}\right)\left(x^{2}+y^{2}\right)-2\left(\alpha^{\prime \prime} c-\alpha c^{\prime \prime}\right) x-2\left(\beta^{\prime \prime} c-\beta c^{\prime \prime}\right) y \\
+c\left(\alpha^{\prime \prime 2}+\beta^{\prime \prime 2}\right)-c^{\prime \prime}\left(\alpha^{2}+\beta^{2}\right)+c^{\prime \prime} c\left(c-c^{\prime \prime}\right)=0 \\
\left(c^{\prime}-c\right)\left(x^{2}+y^{2}\right)-2\left(\alpha c^{\prime}-\alpha^{\prime} c\right) x-2\left(\beta c^{\prime}-\beta^{\prime} c^{\prime}\right) y \\
+c^{\prime}\left(\alpha^{2}+\beta^{2}\right)-c\left(\alpha^{\prime 2}+\beta^{\prime 2}\right)+c c^{\prime}\left(c^{\prime}-c\right)=0
\end{gathered}
$$

and representing these equations by $U=0, U^{\prime}=0, U^{\prime \prime}=0$, we have identically

$$
c U+c^{\prime} U^{\prime}+c^{\prime \prime} U^{\prime \prime}=0,
$$

hence the three tactaction circles pass through the same two points, or what is the same thing, have a common radical axis. 